\title{
Viktimologie, Zeugenschaft und Glaubhaftigkeit
}

\author{
Peer Briken ${ }^{1}$
}

๑ Springer-Verlag GmbH Deutschland, ein Teil von Springer Nature 2019

Die meisten in der forensischen Praxis Tätigen versuchen, die Dynamiken, die bei der Beschäftigung mit „Tätern“ oder „Opfern“ bzw. Betroffenen von Gewalt auftreten, streng auseinanderzuhalten. „Du kannst nicht gleichzeitig mit Tätern und Opfern arbeiten“, heißt es in diesem Zusammenhang oft. An dieser Haltung, die etwas mit Selbstschutz zu tun haben dürfte, scheint also etwas dran zu sein. Kommen die mit beiden Gruppen verbundenen Dynamiken miteinander in Kontakt oder prallen gar aufeinander, so werden dabei meist heftige Affekte freigesetzt: Wut und Empörung über das Unverständnis, mit dem die eine oder andere Seite vertreten wird, Trauer und Enttäuschung über die mangelnde Empathie im gegnerischen Lager, Angst vor dem und Entsetzen über das vielleicht bisher Übersehene oder nicht ausreichend Gewürdigte. Gern wird der eigene Standpunkt beharrlich und auch durchaus machtvoll verteidigt. Unterhalb davon liegt aber vielleicht doch eine Ahnung, die alle gleichzeitig auch schon miteinander teilen: Es könnte mit der Trennung doch nicht so einfach sein. So wie niemand nur gut oder nur böse ist etwas, das viele Kinder über ihre Eltern früh lernen - so ist niemand nur „Täter“ oder „Opfer“. Diejenigen, die mit Menschen arbeiten, die Straftaten begangen haben, kennen deren z.T. massiv mit traumatischen Erfahrungen angefüllten Lebensläufe aus der Rekonstruktion in Gutachten oder dem Versuch der gemeinsamen Reflexion in Therapien, um die Delinquenz mit der Lebensgeschichte in Beziehung zu bringen. Diejenigen, die mit Menschen arbeiten, die von Gewalt betroffen (gewesen) sind, wissen um die Problematik, dass die Entwicklung eigenen gewalttätigen oder aggressiven Verhaltens mit der Betroffenheit korrelieren kann, aber selbstverständlich nicht muss.

Und dennoch entwickeln sich die professionellen Diskurse entlang von Mauern, hinter denen sich Lager mit der

Prof. Dr. med. Peer Briken

briken@uke.de

1 Institut für Sexualforschung, Sexualmedizin \& Forensische Psychiatrie, Universitätsklinikum Hamburg-Eppendorf, Hamburg, Deutschland ihnen eigenen Kultur bilden, vielleicht sich die jeweiligen Mitglieder manchmal sogar verschanzen: hier die „Traumaspezialisten“, da die „Tätertherapeuten“, hier die Opferberatungsstellen für Frauen, da die Beratung gegen Männergewalt - denn, natürlich geht es auch um ein Geschlechterthema, in dem die ausgeübte Gewalt eher männlich und die erlittene eher weiblich verhandelt wird. In Gefängnissen sitzen überwiegend Männer Strafen $a b$, in Frauenhäusern werden Frauen geschützt, auf Borderline-Persönlichkeitsstörung-Spezialstationen werden eher Frauen behandelt, im Maßregelvollzug viel mehr Männer. Das entspricht natürlich der quantitativen Verteilung über die Geschlechter, aber auch das ist wahrscheinlich weit weniger eindeutig, als wir meinen.

Ich schreibe dieses Editorial als jemand, der in mit diesem Jahr neu in den Herausgeberkreis der Zeitschrift Forensische Psychatrie, Psychologie, Kriminologie (FPPK) gekommen ist und für die Zusammenstellung dieses Heftes eine Verantwortung trug. Ich habe zuvor einige Erfahrung als Herausgeber von zwei anderen Zeitschriften und von einigen Büchern gesammelt. Ich habe in diesem Zusammenhang immer wieder auch komplexe Dynamiken erlebt. Ich bin auch im Umfeld meiner Tätigkeit in der Unabhängigen Kommission zur Aufarbeitung sexuellen Kindesmissbrauchs besonders häufig mit Spaltungen konfrontiert - oft werde ich als jemand wahrgenommen, der doch „bisher nur mit Tätern arbeitet ${ }^{1}$, warum nun Aufarbeitung sexuellen Kindesmissbrauchs, bei der es zentral um die Belange der Betroffenen geht ...?"

Dennoch war die Dynamik, die die Vorbereitung dieses Heftes aufgenommen hat, für mich ungewöhnlich, und das hat mit dem Thema zu tun (wenn auch wie immer nicht ausschließlich, sondern selbstverständlich zusätzlich mit Personen, inhaltlicher Qualität von Beiträgen usw.). Es wurde an verschiedenen Stellen sehr emotional. Ich glaube aber, dass die entstandene Dynamik dem Feld guttut: Es gerät dadurch in Bewegung, und, wo Bewegung entsteht,

\footnotetext{
${ }^{1}$ Was im Übrigen nicht stimmt; die ersten Jahre meiner psychiatrischpsychotherapeutischen Tätigkeit arbeitete ich überwiegend mit von Gewalt betroffenen Personen.
} 
kann sich Neues entwickeln, aber selbstverständlich wird dazu auch Altbewährtes infrage gestellt, und das kann zu Verletzungen führen.

Es ist gut, wenn wir die Mauern zwischen den Lagern anbohren, ein paar Gucklöcher zulassen, Spaltungen überwinden, uns selbst Integrationsleistung abverlangen und das Verarbeiten von Affekten. Es dürfte sich lohnen zu sehen, was und wie es die anderen in anderen Lager machen. Auch deswegen ein FPPK-Heft zu Viktimologie, Zeugenschaft und Glaubhaftigkeit.

Zentral in dieser Ausgabe ist der Beitrag von Volbert et al., der einen vor einigen Monaten publizierten Beitrag von Fegert et al. (2018) in der Zeitschrift Nervenheilkunde unter der Überschrift „Enormes professionelles Unverständnis gegenüber Traumatisierten - Ist die Glaubhaftigkeitsbegutachtung und ihre undifferenzierte Anwendung in unterschiedlichen Rechtsbereichen eine Zumutung für von sexueller Gewalt Betroffene?" zum Ausgangspunkt nimmt, um den Wissensstand der aussagepsychologischen Begutachtung darzustellen. Nach Volberts gründlicher Herleitung werden Erinnerungsbeeinträchtigungen, die auch bei tatsächlichem Erlebnisbezug eine Aussage beeinflussen können, in sachgerecht durchgeführten Glaubhaftigkeitsbegutachtungen berücksichtigt. Umgekehrt sei in Glaubhaftigkeitsbegutachtungen aber auch zu prüfen, ob Bedingungen vorgelegen haben, die eine Scheinerinnerung begünstigt haben könnten. Die Berücksichtigung der jeweiligen Beweisschwellen in unterschiedlichen Rechtskontexten sei für aussagepsychologische Gutachten prinzipiell möglich. Schoon und Briken untersuchen die aussagepsychologische Glaubhaftigkeitsbegutachtung im Kontext der Analyse von Zeugenaussagen bei Straftaten gegen die sexuelle Selbstbestimmung unter besonderen Begutachtungsumständen, wie z. B. bei Vorliegen psychischer oder Traumafolgestörungen und kognitiver Einschränkungen aussagender Personen. Wolfs Beitrag beschäftigt sich aus einer juristischen Perspektive mit der Aussagepsychologie und formuliert eine Reihe von Fragen an diese. Leider ist in diesem Beitrag ein Fehler enthalten, der im zugehörigen Erratum entsprechend korrigiert wurde.

Brackmann und Habermeyer zeigen in ihrem Beitrag schließlich einen interkulturellen Vergleich bezüglich der Standards der Befragung von kindlichen Zeugen. Sie erläutern v. a. Erfahrungen aus Großbritannien und den skandinavischen Ländern zur Verbesserung der Qualität von Zeugenaussagen mit einem strukturierten Interviewleitfaden sowie in einem interdisziplinären Kompetenzzentrum zur Befragung von kindlichen Zeugen.

Im Kontext sexuellen Missbrauchs hat es immer wieder tiefe Weggabelungen gegeben, an denen sich Diskurse verändert haben. Mit zwei besonders bedeutsamen Beispielen für den seit 2010 verstärkt in den Blick genommenen sexuellen Missbrauch in Institutionen, beschäftigen sich die bei- den folgenden Arbeiten, der Werkstattbericht aus dem Aufarbeitungsprojekt zur Odenwaldschule von Hackenschmied et al. und eine Arbeit aus der MHG-Studie, deren Veröffentlichung und Reaktionen darauf viele von uns in den letzten Monaten verfolgt haben dürften, bis zu den enttäuschenden Stellungnahmen des Papstes. Die Arbeit von Doelling et al. bestätigt das Ergebnis, dass im Kontext der Missbrauchshandlungen in der katholischen Kirche vielen Beschuldigten nicht nur ein, sondern mehrere Delikte vorgeworfen wurden und viele Betroffene mehrfach einen sexuellen Missbrauch erleiden mussten. Hier schließt später auch Kröbers Blitzlicht an, dass die Änderung der Fallzahlen von sexuellem Missbrauch im Kontext der katholischen Kirche seit den 1990er-Jahren und Gründe diskutiert, warum die Kirche darauf nicht relativierend hinweisen wolle.

Die folgenden Beiträge nähern sich dem Thema Belastungsfaktoren oder eigene Opfererfahrungen bei Personen an, die Straftaten begangen haben. Aebi aus der Züricher forensischen Arbeitsgruppe untersucht mit einer Metaanalyse psychosoziale Belastungen bei jugendlichen Straftätern (z. B. sexueller Missbrauch, Vernachlässigung, psychische und kriminelle Auffälligkeiten in der Familie etc.). Besonders häufig scheinen emotionale Vernachlässigung, Bezeugung von familiärer Gewalt, Trennung der Eltern und Inhaftierung eines Familienmitgliedes auf. Brunner et al. stellen Daten aus der Hamburger Untersuchung von Sexualstraftätern in der Sozialtherapie vor. Hier wurde v.a. der Heimaufenthalt in der Kindheit genauer in den Blick genommen;, ein Thema, das bisher nicht ausreichend untersucht ist, aber wegen struktureller Ähnlichkeiten zwischen Heimerfahrungen und denen in einer Haftanstalt von hoher Bedeutung sein dürfte. Die Gruppe der Sexualstraftäter mit einem Heimaufenthalt in der Kindheit weist eine frühere und stärker delinquente Entwicklung mit einem höheren Rückfallrisiko auf, wobei der Zusammenhang durch den Effekt der traumatisierenden Kindheitserfahrungen vermittelt wird.

$\mathrm{Zu}$ diesen beiden Arbeiten passt der psychiatrische Journal Club von Voulgaris, der eine interessante Längsschnittstudie zum Einfluss von Viktimisierungserfahrungen bei Kindern bzw. Jugendlichen mit sog. sexuellem Problemverhalten untersucht. Es wird deutlich, wie wichtig differenzierte Untersuchungsansätze gerade bei Jugendlichen sind, die eine oberflächliche oder vorschnelle Zuschreibung eines Verhaltens im Sinne der Täterschaft verhindern sollten, um die Entwicklung nicht dadurch zusätzlich negativ zu beeinflussen.

Der freie Beitrag von Beller et al. widmet sich der Frage, inwiefern individuelle religiöse Praktiken, religiöser Fundamentalismus und wahrgenommene religiöse Diskriminierung delinquentes Verhalten von Muslimen prädizieren. Im kriminologischen Journal Club geht es schließlich um die Frage, wie das ,politische Opfersein“ zu definieren und zu 
verstehen ist. Die Entwicklungen im Baskenland und in Nordirland werden dabei als „Fallstudien“ dargestellt.

Ein großer Anteil der Menschen, die Straftaten begangen haben, hat weder mit den eigenen Eltern noch in anderen Bezügen Erfahrungen gemacht, die ihnen eine differenzierte Wahrnehmung von Gut und Böse und den vielen damit verbundenen Zwischenstufen erleichtert hat. Viele Straftäter teilen die Welt in eindeutig Gute und eindeutig Böse ein; alles andere ist ihnen zu unklar, zu unsicher und macht daher Angst. Eine Gesellschaft, die sich in einem differenzierten Umgang mit Aggressionen und Gewalt üben will, muss immer wieder versuchen, simple Spaltungen durch Blicke durch die Mauern zu überwinden und die dabei entstehenden Unsicherheiten aushalten und verarbeiten. Das muss keinesfalls bedeuten, dass wir alle mit Betroffenen und Tätern therapeutisch oder in der Begutachtung arbeiten oder dass wir uns in der Forschung immer beiden Feldern öffnen sollten. Aber es sollte unserer professionellen und toleranten Haltung entsprechen, offen zu sein für den Blick auf die andere Seite.

Interessenkonflikt P. Briken gibt an, dass kein Interessenkonflikt besteht.

\section{Literatur}

Fegert JM, Gerke J, Rassenhofer M (2018) Enormes professionelles Unverständnis gegenüber Traumatisierten. Ist die Glaubhaftigkeitsbegutachtung und ihre undifferenzierte Anwendung in unterschiedlichen Rechtsbereichen eine Zumutung für von sexueller Gewalt Betroffene? Nervenheilkunde 37:525-534 